\title{
Generalized Symmetries and Deformations of the Direct Sums of Lie Algebras
}

\author{
V. D. LyakHovsKy \\ Leningrad State University, Department of Theoretical Physics, Leningrad
}

Received May 16, 1968

\begin{abstract}
The problem of the combined space-time and internal symmetry of elementary particles is investigated from the view-point of the Lie algebras deformation theory. It is demonstrated that the deformations of the direct sum of the Poincare and internal symmetry algebras conserve the structure of the direct sum, if the internal symmetry is semi-simple or is described by the special type of algebras with abelian ideal. So the internal and space-time characteristics of the system remain independent.
\end{abstract}

\section{Introduction}

Difficulties of the group-theoretic description of elementary particles are often connected with non-relativistic character of usually used internal symmetry groups. This forced the attempts of the joint description of the space-time and internal properties. If the inhomogeneous Lorentz group, which is supposed to be a subgroup of the generalized symmetry group, is its direct factor, all the particles belonging to the internal symmetry multiplet must have the same mass. All the deviations from this simplest scheme have not yet given positive results. The so called no-go theorems are proved within quite general suppositions $[1-5]$. In this connection it is interesting to consider a certain class of Lie algebras, in which the structure of the direct sum and Poincare subalgebra appear only after the contraction [6-9]. First of all they would not contain Poincaré subalgebra before the limiting transition and no-go theorems would be unapplicable to them. On the other hand after the contraction they would turn into the direct sum $P \oplus A$ so that the physical interpretation of their operators would be possible. The existence of the limiting transition indicates the closeness [6] of the initial and contracted algebras. Experimental errors often make close algebras undistinguishable. So if one is not satisfied with the initial algebra it is possible to substitute it by the close one. Thus the problem of the relativization stimulates the search for the algebras that are close to the direct sum $P \oplus A$. Were they found, such algebras would be useful for the investigation of the physical spaces [13] and mass spectra of elementary particles [10-12].

Using the contraction procedure it is impossible to reconstruct all the Lie algebras which form the neighbourhood of the given algebra. The 
effective method to find all such algebras, based on the theory of deformations of Lie algebras [14], was proposed by LEvY-NAHAS [15]. Here the deformations of $P \oplus A$-type algebras are investigated. The methods used depend essentially on the properties of algebra $A$. Deformations of $P \oplus S$ algebra, where the internal symmetry algebra $S$ is semi-simple are considered in $\S 2$. In $\S 3$ the special class of internal symmetry algebras $A=S \oplus I$ with abelian ideal $I$ is studied. These examples describe almost all physically interesting cases. It is proved that all deformed algebras conserve the structure of the direct sum. Space-time and internal characteristics of the system remain thus entirely independent, so that it is impossible to construct physically interesting generalized symmetry.

\section{Semi-Simple Internal Symmetry}

Let the internal symmetry of the system be described by the semisimple algebra $S$. What are then the deformations of the direct sum $P \oplus S$ ?

We shall first suppose, that the dimension of the 3-cohomology group is equal to zero.

$$
\operatorname{dim} H^{3}(P \oplus S, P \oplus S)=0
$$

Then the number of non-trivial un-equivalent deformations of the first order coincides with the dimension of the 2-cohomology group $H^{2}(P \oplus S$, $P \oplus S)$. For calculations we use Serré and Hochschild's formula [16]

$$
H^{n}(G, M)=\sum_{i+j=n} H^{i}\left(\frac{G}{K}, F\right) \bigotimes_{F} H^{j}(K, M)^{G}
$$

where $M$ is a free $G$-modul,

$K$ is an ideal of $G$-algebra such, that $G / K$ is semi-simple,

$F$ is the ring over which $G$ is defined,

$H^{j}(K, M)^{G}$ is the group of $G$-invariant elements in $H^{j}(K, M)$.

Let us write the decomposition $P=T \dot{\oplus} L$ for the Poincaré algebra. For this case the 2-cohomology group may be simplified

$$
H^{2}(P \oplus S, P \oplus S)=H^{2}(T, P \oplus S)^{P \oplus S}
$$

Suppose $\varrho$ denotes the map $T \stackrel{\varrho}{\longrightarrow} P \oplus S$. Then $b \in B^{2}(T, P \oplus S)$, if and only if

$$
b\left(t_{i}, t_{k}\right)=\left[t_{i}, \varrho\left(t_{k}\right)\right]-\left[t_{k}, \varrho\left(t_{i}\right)\right] .
$$

As it was shown in [15] the condition (4) is satisfied for all maps $\varphi$ of $T \wedge T$ into $T$. Thus $B^{2}(T, P \oplus S)=\operatorname{Hom}_{2}(E(T), T)$, where $E(T)$ is an exterior algebra of $K$-modul $T$. 
Now, the 2-coboundaries being defined, we can use instead of the $P \oplus S$-invariance condition for the elements $\eta \in H^{2}(T, P \oplus S)$

$$
\begin{aligned}
& (a \cdot \eta)\left(t_{i}, t_{k}\right) \equiv\left[a, \eta\left(t_{i}, t_{k}\right)\right]-\eta\left(\left[a, t_{i}\right], t_{k}\right)-\eta\left(t_{i},\left[a, t_{k}\right]\right)=0 \\
& a \in P \oplus S
\end{aligned}
$$

the corresponding relation for the cocycles $\varphi \in Z^{2}(T, P \oplus S)$

$$
(a \cdot \varphi)\left(t_{i}, t_{k}\right) \in B^{2}(T, P \oplus S)=\operatorname{Hom}_{2}(E(T), T) .
$$

Before we find out what are the homomorphisms $T \wedge T \rightarrow P \oplus S$ that form the group $Z^{2}(T, P \oplus S)$, let us investigate the relation (6) for all the elements $f \in \mathrm{Hom}_{2}(E(T), P \oplus S)$. According to the domain of values let $f$ be represented in the form $f=f_{T}+f_{L}+f_{S}$. It is not difficult to show that in addition to $f_{T} \in \operatorname{Hom}_{2}(E(T), T)=B^{2}(T, P \oplus S)$ the relation (6) is satisfied also for $f_{L}$ and $f_{S}$ such that

$$
\begin{aligned}
{\left[l, f_{L}\left(t_{i}, t_{k}\right)\right]-f_{L}\left(\left[l, t_{i}\right], t_{k}\right)-f_{L}\left(t_{i},\left[l, t_{k}\right]\right) } & =0, \\
{\left[s, f_{S}\left(t_{i}, t_{k}\right)\right] } & =0,
\end{aligned}
$$

where $l \in L, s \in S$. It follows from the semi-simplicity of the algebra $S$, that Eq. (8) is true only for zero map $f_{S}$.

Finally the dimension of $H^{2}(T, P \oplus S)^{P \oplus S}$ and the first order deformations of the Lie algebra $P \oplus S$ (the condition (6) being fulfilled) are entirely defined by those linearly independent functions that satisfy the Eq. (6) and belong to $Z^{2}(T, P \oplus S)$. Inasmuch as both the arguments and the values of these functions are in $P$, the deformations connected with them may only change the Poincaré algebra and cannot deform the internal symmetry or modify the structure of the direct sum. Such deformations are of no physical interest.

In that case the investigation of $H^{3}(P \oplus S, P \oplus S)$ becomes unnecessary. For if $\operatorname{dim} H^{3}(P \oplus S, P \oplus S) \neq 0$, then the number of nontrivial deformations may only decrease compared with $\operatorname{dim} H^{2}(P \oplus S$, $P \oplus S)$.

As for the deformations of the higher orders all of them are trivial. Indeed, the conditions obtained by LEVY-NAHAS [15] in the case of the Poincaré algebra coincide with those presented by the Eq. (7) and the requirement $f_{L} \in Z^{2}(T, P \oplus S)$. For the Poincaré algebra there are only two infinitesimal deformations, these are the simple algebras $S O(1,4)$ and $S O(2,3)$. Hence the direct sums $S O(1,4) \oplus S$ and $S O(2,3) \oplus S$ are the only first order deformations of the $P \oplus S$ algebra. It follows from their semi-simplycity that the next order deformations are trivial.

Whatever semi-simple algebra one takes to describe the internal symmetry, the deformation theory does not give the possibility to construct algebra close to $P \oplus S$. 


\section{The Special Case of Internal Symmetry}

Let us try to obtain physically non-trivial deformations via complication of the internal symmetry algebra. Suppose now $A=S \dot{\oplus} I$, where $S$ is simple and $I$ is abelian ideal. Suppose also that

$$
\operatorname{dim} H^{3}(P \oplus A, P \oplus A)=0 .
$$

We are interested in the 2-cohomology group $H^{2}(P \oplus A, P \oplus A)$ which may be calculated using the Eq. (2)

$$
H^{2}(P \oplus A, P \oplus A)=H^{2}(T \oplus I, P \oplus A)^{P \oplus A} .
$$

First let us find the 2-coboundaries $B^{2}(T \oplus I, P \oplus A)$. As well as for a semi-simple internal symmetry $(\S 2)$ all the elements of $\mathrm{Hom}_{2}(E(T), T)$ are coboundaries. Besides that the elements of $\operatorname{Hom}_{2}(E(I), I)$ may also belong to $B^{2}(T \oplus I, P \oplus A)$. To avoid cumbersome calculations, we shall consider that $B^{2}(T \oplus I, P \oplus A)$ contains all the homomorphisms of $\mathrm{Hom}_{2}(E(I), I)$. This leads to certain restrictions on the structure of $A$.

All necessary algebras $A$ can be obtained by means of $I W$-contraction of Lie algebras corresponding to the $S O(m, n)$ groups. If $L_{p q}$ is their standard Lie basis, then the contraction should be such that a set of elements $L_{p q}$ with $q$ fixed form an abelian ideal ( $p=1,2,3, \ldots, q-1$, $q+1, \ldots, m+n-1)$.

If we consider now only these algebras of internal symmetry, every element of $\mathrm{Hom}_{2}(E(I), I)$ will be a coboundary.

The elements of $\operatorname{Hom}(I \wedge T, I \oplus T)$ form the third set of the coboundaries. By definition the map $b_{T \oplus I}\left(i_{k}, t_{n}\right)$ belongs to $B^{2}(T \oplus I, P \oplus A)$ if and only if

$$
b_{T \oplus I}\left(i_{k}, t_{n}\right)=\left[i_{k}, \varrho\left(t_{n}\right)\right]-\left[t_{n}, \varrho\left(i_{k}\right)\right],
$$

where $\varrho$ is the homomorphism $T \oplus I \rightarrow P \oplus A$. Let us write the functions $b$ and $\varrho$ in the following form

$$
\begin{aligned}
b_{T \oplus I}\left(i_{k}, t_{n}\right) & =\beta_{k n}^{j} i_{j}+\beta_{k n}^{l} t_{l}, \\
\varrho_{L}\left(i_{k}\right) & =d_{k}^{u} l_{u}, \\
\varrho_{S}\left(t_{n}\right) & =d_{n}^{v} s_{v} .
\end{aligned}
$$

where $i, t, l$ and $s$ are the basis elements of subalgebras $I, T, L$ and $S$ respectively. Let $C$ be the structure constants of $P \oplus A$, then it follows from the Eq. (10) that

$$
\begin{aligned}
& \beta_{k n}^{j}=C_{k v}^{j} d_{n}^{v} \\
& \beta_{k n}^{l}=C_{n u}^{l} d_{k}^{u} .
\end{aligned}
$$

Only those homomorphisms of $\operatorname{Hom}(I \wedge T, I \oplus T)$ are coboundaries which have in the decomposition (11) coefficients satisfying the Eq. (12). 
Now we may investigate $P \oplus A$-invariance for the elements $\varphi\left(j_{1}, j_{2}\right)$ of $Z^{2}(T \oplus I, P \oplus A)$

$$
\begin{gathered}
{\left[\eta, \varphi\left(j_{1}, j_{2}\right)\right]-\varphi\left(\left[\eta, j_{1}\right], j_{2}\right)-\varphi\left(j_{1},\left[j_{2}, \eta\right]\right) \in B^{2}(T \oplus I, P \oplus A)} \\
\eta \in P \oplus A ; j \in T \oplus I .
\end{gathered}
$$

For the same reasons as those mentioned in $\S 2$ it is convenient to study first the invariance of the whole group $\operatorname{Hom}_{2}(E(T \oplus I), P \oplus A)$ and then to search for 2-cocycles.

Functions $f_{T}\left(t_{1}, t_{2}\right)$ and $f_{I}\left(i_{1}, i_{2}\right)$, the elements of $\operatorname{Hom}_{2}(E(T), T)$ and $\operatorname{Hom}_{2}(E(I), I)$ respectively, are evidently invariant.

It follows from the Eq. (13) that the maps $f_{T}(t, i)$ and $f_{I}(t, i)$ are $T \oplus I$-invariant. But only two sets of these functions are $L$-invariant. The first one consists of the functions belonging to $B^{2}(T \oplus I, P \oplus A)$, i.e. satisfying Eq. (11) and (12). The second set contains those maps for which $\beta_{k n}^{k}$ and $\beta_{k n}^{n}$ are the only non-zero coefficients in the decomposition (11) $\left(\beta_{k n}^{k}\right.$ are equal for $n$ fixed and this is also true for all $\beta_{k n}^{n}$ with $k$ fixed). Among these two $L$-invariant sets only the first is $S$-invariant. That is, functions $f_{T \oplus I}(t, i)$ are $P \oplus A$-invariant only if they are 2-coboundaries.

For the homomorphisms $f_{L}\left(t_{1}, t_{2}\right)$ only $L$-invariance is essential thus we have

$$
\left[l_{r}, f_{L}\left(t_{k}, t_{n}\right)\right]-f_{L}\left(\left[l_{r}, t_{k}\right], t_{n}\right)-f_{L}\left(t_{k},\left[l_{r}, t_{n}\right]\right)=0 .
$$

Similarly, $f_{S}\left(i_{k}, i_{n}\right)$ are $P \oplus A$-invariant if they satisfy the following equations

$$
\left[s_{r}, f_{S}\left(i_{k}, i_{n}\right)\right]-f_{S}\left(\left[s_{r}, i_{k}\right], i_{n}\right)-f_{S}\left(i_{k},\left[s_{r}, i_{n}\right]\right)=0 .
$$

It is not necessary to verify the invariance of $f_{L}(t, i)$ and $f_{S}(t, i)$, for, as we shall see later they are not the 2-cocycles.

Indeed, using the explicit form of the condition $f_{L \oplus S}(t, i) \in Z^{2}(T \oplus I$, $P \oplus A$ ) we obtain finally the equations

$$
\begin{aligned}
& {\left[t_{m}, f_{L}\left(t_{k}, i\right)\right]-\left[t_{k}, f_{L}\left(t_{m}, i\right)\right]=0,} \\
& {\left[i_{m}, f_{S}\left(t, i_{k}\right)\right]-\left[i_{k}, f_{S}\left(t, i_{m}\right)\right]=0 .}
\end{aligned}
$$

If the parameters in (16) and (17) ( $i$ and $t$ respectively) are fixed one may consider functions $f_{L}$ and $f_{S}$ to be the homomorphisms $T \rightarrow L$ and $I \rightarrow S$ and the Eqs. (16) and (17) have then the meaning of $\delta f_{L}=0$ and $\delta f_{S}=0$. On the other hand it follows from the structure of $P$ and $A$ that $f_{L} \ddagger Z^{1}(T, P)$ and $f_{S} \notin Z^{1}(I, A)$. Hence the Eqs. (16) and (17) have no other solutions than zero and $f_{L}(t, i), f_{S}(t, i)$ do not belong to $Z^{2}(T \oplus I$, $P \oplus A)$. Thus their invariance is of no importance. 
Among the invariant functions only $f_{L}(t, t)$ and $f_{S}(i, i)$ are not cohomologous to zero. They are 2-cocycles if the following relations are realized

$$
\begin{aligned}
\sum_{P(n, m, k)}\left[t_{n}, f_{L}\left(t_{m}, t_{k}\right)\right] & =0, \\
\sum_{P(n, m, k)}\left[i_{n}, f_{S}\left(i_{m}, i_{k}\right)\right] & =0,
\end{aligned}
$$

where $P(n, m, k)$ is the circular permutation of $n, m$ and $k$.

Finally, all essential first order deformations are described by the solutions of the Eq. (14), (15), (16) and (17). But all of them have such arguments and values that the corresponding deformations cannot change the structure of the direct sum in the initial algebra. Therefore the minimal complication of the internal symmetry algebra studied in this section do not help to discover physically applicable close algebras, when the first order deformations are considered.

Just as in the previous case the condition $\operatorname{dim} H^{3}(P \oplus A, P \oplus A)=0$ becomes unimportant.

The higher order deformations may be non-trivial only if in the first order we do not obtain semi-simple algebra. The solutions of the Eq. (14) and (16) perform the deformation of the Poincaré algebra into simple De-Sitter algebras [15]. In the general case the Eq. (15) has the unique solution $f_{S}\left(i_{k}, i_{n}\right) \sim S_{k n}$, where $S_{k n}$ is the generator of rotations in the $k n$-plane. This function is the 2-cocycle, i.e. (17) is satisfied. Only for algebras $A$ of dimension 10 (the Poincaré algebra is among them) there is an exception. In this case (15) has two solutions but one of them is not the 2-cocycle. Therefore the system of the Eq. (15) and (17) always has the unique solution and the first order deformations of the algebra $A$ are 1-dimensional. On the other hand there must be a deformation of $A$ which is a procedure inverse to the $I W$-contraction that generates $A$. That means that the deformations of the first order transform $A$ into the simple algebra that was contracted to obtain $A$.

If the deforming function contains both $f_{L}(t, t)$ and $f_{S}(i, i)$, the first order deformation gives the semi-simple algebras $S O(1,4) \oplus S O(m, n)$ or $S O(2,3) \oplus S O(m, n)$. If we take $f_{S}(i, i)$ alone, the result will be $P \oplus S O(m, n)$. As it follows from $\S 2$ the deformations of this algebra are $S O(1,4) \oplus S O(m, n)$ or $S O(2,3) \oplus S O(m, n)$. Using $f_{L}(t, t)$ as the deforming function one obtains algebras $S O(1,4) \oplus A$ or $S O(2,3) \oplus A$. The results similar to those of $\S 1$ can be very easily obtained for these algebras and that makes evident that the only possible deformations are $S O(1,4) \oplus S O(m, n)$ or $S O(2,3) \oplus S O(m, n)$. In all cases the algebras obtained are semi-simple, and the higher order deformations are trivial. 


\section{Literature}

1. PaIs, A.: Rev. Mod. Phys. 38, 215 (1966).

2. Flato, M., and D. Sternheimer: Phys. Rev. Letters 19, 254 (1967).

3. Roskies, R.: J. Math. Phys. 7, 395 (1966).

4. Fleischman, O., and J. G. Nagel: J. Math. Phys. 8, 1128 (1967).

5. Stern, J.: Nuovo Cimento IL A, 33 (1967).

6. Segal, I. E.: Duke Math. J. 18, 221 (1951).

7. Inönü, E., and E. P. Wigner: Proc. Nat. Acad. Sci. 39, 510 (1953).

8. Saletan, E. J.: J. Math. Phys. 2, 1 (1961).

9. Doebiner, H. D., and O. Melsheimer: Nuovo Cimento IL A, 306 (1967).

10. Barut, A. O., and A. Böнm: Phys. Rev. 139 B, 1107 (1965).

11. - Phys. Rev. 145, 1212 (1966).

12. 一, and H. Kleinert: Phys. Rev. 157, 1180 (1967).

13. GÜRSEY, F.: Group theoretical concepts and methods in elementary particle physics. Istanbul Summer School Lectures, p. 365.

14. Gerstenhaber, M.: Ann. Math. 79, 59 (1964).

15. Levy-Nahas, M.: J. Math. Phys. 8, 1211 (1967).

16. Serré, J. P., and G. Hochschild : Ann. Math. 57, 591 (1953).

V. D. Lyakhovsky

Department of Theoretical Physics

Leningrad State University

Leningrad, B-164, USSR 